Pacific

Journal of

Mathematics

THE NEF CONES OF AND MINIMAL-DEGREE CURVES IN THE HILBERT SCHEMES OF POINTS ON CERTAIN SURFACES

Zhenbo QIN AND YUPING TU 


\title{
THE NEF CONES OF AND MINIMAL-DEGREE CURVES IN THE HILBERT SCHEMES OF POINTS ON CERTAIN SURFACES
}

\author{
ZHENBO QIN AND YUPING TU
}

\begin{abstract}
We determine the nef cones of the Hilbert schemes of points on certain surfaces $X$ with $h^{1}\left(X, \mathcal{O}_{X}\right)=0$. Then we apply the results to Hirzebruch surfaces, and study the minimal-degree curves in the Hilbert schemes of points on Hirzebruch surfaces. Our results generalize those in Li, Qin, and Zhang (2003).
\end{abstract}

\section{Introduction}

Hilbert schemes are classical objects in algebraic geometry, and have been studied extensively since their constructions by Grothendieck. Hilbert schemes of points on smooth surfaces are known to be smooth and irreducible, and have deep connections with combinatorics, representation theory and string theory. Ample divisors on these Hilbert schemes were considered in [Beltrametti and Sommese 1991; 1993; Catanese and Gœttsche 1990]. The nef cones of the Hilbert schemes of points on smooth surfaces were first investigated in [Li et al. 2003] when the surface is the projective plane. Recently, these nef cones were further understood in [Arcara et al. 2013; Bertram and Coskun 2013; Bolognese et al. 2015] via Bridgeland stability.

In this paper, we generalize the methods and results in [Li et al. 2003], and prove a structure theorem for the nef cones of the Hilbert schemes of points on certain surfaces. To state our result, let $X$ be a smooth projective complex surface. The nef cone of $X$ is the span of the nef divisors on $X$. We use $\mathrm{NE}(X)$ to denote the cone spanned by all the effective curves in $X$. It is well-known that $\mathrm{NE}(X)$ is dual to the nef cone of $X$. Let $X^{[n]}$ be the Hilbert scheme of points in $X$. By [Fogarty 1968; Iarrobino 1977], $X^{[n]}$ is a smooth irreducible variety of dimension $2 n$.

Theorem 1.1. Let $n \geq 2$, and let the surface $X$ satisfy $h^{1}\left(X, \mathcal{O}_{X}\right)=0$. Assume that the nef cone of $X$ is the span of the divisors $F_{1}, \ldots, F_{t}$, and the cone $\mathrm{NE}(X)$ is the

Qin partially supported by a grant from the Simons Foundation. Tu partially supported by NSFC grant 11171258.

MSC2010: 14C05.

Keywords: nef cones, Hilbert schemes. 
span of the curves $C_{1}, \ldots, C_{t}$ with $F_{i} \cdot C_{j}=\delta_{i, j}$ for all $i$ and $j$. Assume further that

$$
\mathcal{O}_{X}\left((n-1) \sum_{i=1}^{t} F_{i}\right)
$$

is $(n-1)$-very ample. Then

(i) the nef cone of the Hilbert scheme $X^{[n]}$ is spanned by

$$
D_{F_{1}}, \ldots, D_{F_{t}},(n-1) \sum_{i=1}^{t} D_{F_{i}}-B_{n} / 2 ;
$$

(ii) the cone $\mathrm{NE}\left(X^{[n]}\right)$ is spanned by the classes

$$
\beta_{C_{1}}-(n-1) \beta_{n}, \ldots, \beta_{C_{t}}-(n-1) \beta_{n}, \beta_{n} .
$$

In the above theorem, $B_{n}$ denotes the boundary divisor of the Hilbert scheme $X^{[n]}$ consisting of the elements $\xi \in X^{[n]}$ which are not smooth as subschemes of $X^{[n]}$, and $\beta_{n}$ is the minimal curve class contracted by the Hilbert-Chow morphism $X^{[n]} \rightarrow X^{(n)}$ sending an element $\xi \in X^{[n]}$ to its support (with multiplicities) in the $n$-th symmetric product $X^{(n)}$ of $X$. We refer to (2-4), (2-3) and Definition 2.1 for the definitions of $D_{F}, \beta_{C}$ and $(n-1)$-very ampleness, respectively. Theorem 1.1 is proved in Section 2. Our main idea in the proof of Theorem 1.1 is to construct curves in $X^{[n]}$ which provide us with information about the nef divisors in $X^{[n]}$.

In Section 3, we apply Theorem 1.1 to the case when $X$ is a Hirzebruch surface, and recover a result in [Bertram and Coskun 2013]. Moreover, when $X$ is a Hirzebruch surface, we classify all the curves in the Hilbert scheme $X^{[n]}$ whose homology classes are contained in the list (1-2). These curves have minimal degree in the sense that their intersection numbers with certain very ample divisors in $X^{[n]}$ are all equal to 1 . We compute the normal bundles of these curves, and prove that their moduli spaces are unobstructed, i.e., are smooth with the expected dimensions.

Conventions. Let $0 \leq k \leq n$ and $V$ be an $n$-dimensional vector space. We use the Grassmannian $\mathbb{G}(V, k)$ to denote the set of all $k$-dimensional quotients of $V$, or equivalently, the set of all $(n-k)$-dimensional subspaces of $V$. Also, we take $\mathbb{P}(V)=\mathbb{G}(V, 1)$. So the set of lines in $\mathbb{P}(V)$ is the Grassmannian $\mathbb{G}(V, 2)$.

\section{The nef cones of the Hilbert schemes of points on certain surfaces}

In this section, we study the nef cones of the Hilbert schemes of points on certain surfaces with $h^{1}\left(X, \mathcal{O}_{X}\right)=0$. Our goal is to prove Theorem 1.1.

Let $X$ be a smooth projective complex surface, and $X^{[n]}$ be the Hilbert scheme of points in $X$. An element in $X^{[n]}$ is represented by a length- $n 0$-dimensional closed 
subscheme $\xi$ of $X$. For $\xi \in X^{[n]}$, let $I_{\xi}$ be the corresponding sheaf of ideals and $\mathcal{O}_{\xi}$ the structure sheaf. The subset

$$
B_{n}=\left\{\xi \in X^{[n]}|| \operatorname{Supp}(\xi) \mid<n\right\}
$$

is defined to be the boundary of $X^{[n]}$. Let $C$ be a real surface in $X$, and fix distinct points $x_{1}, \ldots, x_{n-1} \in X$ which are not contained in $C$. Define

$$
\begin{aligned}
\beta_{n} & =\left\{\xi+x_{2}+\cdots+x_{n-1} \in X^{[n]} \mid \operatorname{Supp}(\xi)=\left\{x_{1}\right\}\right\}, \\
\beta_{C} & =\left\{x+x_{1}+\cdots+x_{n-1} \in X^{[n]} \mid x \in C\right\}, \\
D_{C} & =\left\{\xi \in X^{[n]} \mid \operatorname{Supp}(\xi) \cap C \neq \varnothing\right\} .
\end{aligned}
$$

Note that $\beta_{C}$ is a curve, and $D_{C}$ a divisor, in $X^{[n]}$ when $C$ is a smooth algebraic curve in $X$. We extend the notions $\beta_{C}$ and $D_{C}$ to all the divisors $C$ in $X$ by linearity.

Nakajima [1997] and Grojnowski [1996] geometrically constructed a Heisenberg algebra action on the cohomology of the Hilbert schemes $X^{[n]}$. Let $H^{*}(X)$ be the total cohomology of $X$ with $\mathbb{C}$-coefficients. Denote the Heisenberg operators of Nakajima and Grojnowski by $\mathfrak{a}_{m}(\alpha)$ where $m \in \mathbb{Z}$ and $\alpha \in H^{*}(X)$. Set

$$
\mathbb{H}_{X}=\bigoplus_{n=0}^{+\infty} H^{*}\left(X^{[n]}\right) .
$$

Then the space $\mathbb{H}_{X}$ is an irreducible representation of the Heisenberg algebra generated by the operators $\mathfrak{a}_{m}(\alpha)$ with the highest weight vector being

$$
|0\rangle=1 \in H^{*}\left(X^{[0]}\right)=\mathbb{C} .
$$

It follows that the $n$-th component $H^{*}\left(X^{[n]}\right)$ in the Fock space $\mathbb{H}_{X}$ is linearly spanned by the Heisenberg monomial classes

$$
\mathfrak{a}_{-n_{1}}\left(\alpha_{1}\right) \cdots \mathfrak{a}_{-n_{k}}\left(\alpha_{k}\right)|0\rangle,
$$

where $k \geq 0, n_{1}, \ldots, n_{k}>0$ and $n_{1}+\cdots+n_{k}=n$. We have

$$
\begin{aligned}
\beta_{n} & =\mathfrak{a}_{-2}(x) \mathfrak{a}_{-1}(x)^{n-2}|0\rangle, \\
\beta_{C} & =\mathfrak{a}_{-1}(C) \mathfrak{a}_{-1}(x)^{n-1}|0\rangle, \\
B_{n} & =\frac{1}{(n-2) !} \mathfrak{a}_{-1}\left(1_{X}\right)^{n-2} \mathfrak{a}_{-2}\left(1_{X}\right)|0\rangle, \\
D_{C} & =\frac{1}{(n-1) !} \mathfrak{a}_{-1}\left(1_{X}\right)^{n-1} \mathfrak{a}_{-1}(C)|0\rangle,
\end{aligned}
$$

where $x$ and $1_{X}$ denote the cohomology classes corresponding to a point $x \in X$ and the surface $X$, respectively. Abusing notation, we also use $C$ to denote the cohomology class corresponding to the real surface $C$. 
The following important definition is from [Beltrametti and Sommese 1991].

Definition 2.1. Let $n \geq 1$. A line bundle $L$ on the surface $X$ is $(n-1)$-very ample if the restriction $H^{0}(X, L) \rightarrow H^{0}\left(X, \mathcal{O}_{\xi} \otimes L\right)$ is surjective for every $\xi \in X^{[n]}$.

The concept of $(n-1)$-very ampleness relates $X^{[n]}$ to a Grassmannian as follows. The surjective map in Definition 2.1 represents an element in $\mathbb{G}\left(H^{0}(X, L), n\right)$. So if $L$ is $(n-1)$-very ample, then we obtain a morphism

$$
\varphi_{n}(L): X^{[n]} \rightarrow \mathbb{G}\left(H^{0}(X, L), n\right) .
$$

Let $h=h^{0}(X, L)$, and let $\mathfrak{P}: \mathbb{G}\left(\mathbb{C}^{h}, n\right) \rightarrow \mathbb{P}\left(\left(\bigwedge^{h-n} \mathbb{C}^{h}\right)^{*}\right)$ be the Plücker embedding. Then we see from the Appendix of [Beltrametti and Sommese 1991] that

$$
\left(\mathfrak{P} \circ \varphi_{n}(L)\right)^{*} \mathcal{H}=\mathcal{O}_{X^{[n]}}\left(D_{c_{1}(L)}-B_{n} / 2\right),
$$

where $\mathcal{H}$ is the hyperplane line bundle over the projective space $\mathbb{P}\left(\left(\bigwedge^{h-n} \mathbb{C}^{h}\right)^{*}\right)$.

Lemma 2.2. If $L$ is $(n-1)$-very ample, then the divisor $D_{c_{1}(L)}-B_{n} / 2$ is nef. If $L$ is $n$-very ample, then the divisor $D_{c_{1}(L)}-B_{n} / 2$ is very ample.

The first statement in Lemma 2.2 follows immediately from (2-10), and the second statement was proved in [Catanese and Gœttsche 1990].

In $X^{[n]} \times X$, we have the universal codimension-2 subscheme

$$
\mathcal{Z}_{n}=\left\{(\xi, x) \in X^{[n]} \times X \mid x \in \operatorname{Supp}(\xi)\right\} \subset X^{[n]} \times X .
$$

Define the incidence variety $X^{[n-1, n]}=\left\{(\xi, \eta) \in X^{[n-1]} \times X^{[n]} \mid \xi \subset \eta\right\}$. It is wellknown [Cheah 1998; Tikhomirov 1994] that $X^{[n-1, n]}$ is smooth and of dimension $2 n$. Define

$$
\begin{array}{ll}
f_{n}: X^{[n-1, n]} \rightarrow X^{[n-1]} & \text { with } f_{n}(\xi, \eta)=\xi, \\
g_{n}: X^{[n-1, n]} \rightarrow X^{[n]} & \text { with } g_{n}(\xi, \eta)=\eta, \\
\rho: X^{[n-1, n]} \rightarrow X & \text { with } \rho(\xi, \eta)=\operatorname{Supp}\left(I_{\xi} / I_{\eta}\right) .
\end{array}
$$

Set $\phi_{n}=\left(f_{n}, \rho\right): X^{[n-1, n]} \rightarrow X^{[n-1]} \times X$. By Proposition 2.2 in [Ellingsrud and Strømme 1998], $\phi_{n}$ is the blowing-up morphism of $X^{[n-1]} \times X$ along $\mathcal{Z}_{n-1}$.

Next, let $C$ be an irreducible curve in $X$. Let $\xi=x_{1}+\cdots+x_{n-1} \in X^{[n-1]}$, where $x_{1}, \ldots, x_{n-1}$ are distinct smooth points on $C$. Let $(C+\xi)$ be the closure of $(C-\operatorname{Supp}(\xi))+\xi$ in $X^{[n]}$. Alternatively, consider

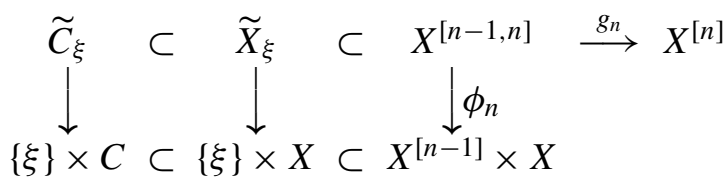

where $\widetilde{C}_{\xi}$ and $\widetilde{X}_{\xi}$ are the strict transforms of $\{\xi\} \times C$ and $\{\xi\} \times X$ in $X^{[n-1, n]}$, respectively. Since $\phi_{n}$ is the blowing-up morphism of $X^{[n-1]} \times X$ along $\mathcal{Z}_{n-1}$, it 
follows that $\widetilde{X}_{\xi}$ is isomorphic to the blowup of $\{\xi\} \times X \cong X$ at $x_{1}, \ldots, x_{n-1}$. For $1 \leq i \leq(n-1)$, let $E_{i}$ be the exceptional divisor in $\widetilde{X}_{\xi}$ over $x_{i}$. Then we obtain

$$
\left(\phi_{n} \mid \widetilde{X}_{\xi}\right)^{*}(\{\xi\} \times C)=\widetilde{C}_{\xi}+\sum_{i=1}^{n-1} E_{i}
$$

in the Chow group $A_{1}\left(\widetilde{X}_{\xi}\right)$. Notice that $g_{n}\left(\widetilde{C}_{\xi}\right)=(C+\xi)$ and

$$
g_{n}\left(E_{i}\right)=M_{2}\left(x_{i}\right)+x_{1}+\cdots+x_{i-1}+x_{i+1}+\cdots+x_{n-1} .
$$

In fact, since $\left.g_{n}\right|_{\widetilde{X}_{\xi}}: \widetilde{X}_{\xi} \rightarrow g_{n}\left(\widetilde{X}_{\xi}\right)$ is an isomorphism, we have

$$
\left(\left.g_{n}\right|_{\tilde{X}_{\xi}}\right)_{*}\left(\widetilde{C}_{\xi}\right)=(C+\xi) \text { and }\left(\left.g_{n}\right|_{\widetilde{X}_{\xi}}\right)_{*}\left(E_{i}\right)=\beta_{n} .
$$

Lemma 2.3. With the above notation, $(C+\xi)=\beta_{C}-(n-1) \beta_{n}$ in $A_{1}\left(X^{[n]}\right)$.

Proof. Choose two smooth curves $C_{1}$ and $C_{2}$ in $X$ such that $C=C_{1}-C_{2}$ in $A_{1}(X)$ and $\operatorname{Supp}(\xi) \cap\left(C_{1} \cup C_{2}\right)=\varnothing$. Then in $A_{1}\left(X^{[n]}\right)$, we have

$$
\begin{aligned}
\left(g_{n} \mid \widetilde{X}_{\xi}\right)_{*}\left(\phi_{n} \mid \tilde{X}_{\xi}\right)^{*}(\{\xi\} \times C) & \\
& =\left(g_{n} \mid \tilde{X}_{\xi}\right)_{*}\left(\phi_{n} \mid \widetilde{X}_{\xi}\right)^{*}\left(\{\xi\} \times C_{1}\right)-\left(g_{n} \mid \widetilde{X}_{\xi}\right)_{*}\left(\phi_{n} \mid \tilde{X}_{\xi}\right)^{*}\left(\{\xi\} \times C_{2}\right) \\
& =\left(C_{1}+\xi\right)-\left(C_{2}+\xi\right) \\
& =\beta_{C_{1}}-\beta_{C_{2}}=\beta_{C} .
\end{aligned}
$$

On the other hand, applying (2-13) and (2-14), we conclude that

$$
\left(\left.g_{n}\right|_{\widetilde{X}_{\xi}}\right)_{*}\left(\left.\phi_{n}\right|_{\widetilde{X}_{\xi}}\right)^{*}(\{\xi\} \times C)=\left(\left.g_{n}\right|_{\widetilde{X}_{\xi}}\right)_{*}\left(\widetilde{C}_{\xi}+\sum_{i=1}^{n-1} E_{i}\right)=(C+\xi)+(n-1) \beta_{n} .
$$

Combining this with (2-15), we see that $(C+\xi)=\beta_{C}-(n-1) \beta_{n}$ in $A_{1}\left(X^{[n]}\right)$.

Lemma 2.4. Let $F$ be a divisor on $X$. If $D_{F}-d\left(B_{n} / 2\right)$ is nef, then $d \geq 0$ and $F \cdot C \geq d(n-1)$ for every irreducible curve $C \subset X$. In particular, $F$ is nef. Proof. Note that $D_{F} \cdot \beta_{n}=0$ and $B_{n} \cdot \beta_{n}=-2$. Thus, we have

$$
0 \leq\left(D_{F}-d\left(B_{n} / 2\right)\right) \cdot \beta_{n}=d .
$$

Since $D_{F} \cdot \beta_{C}=F \cdot C$ and $B_{n} \cdot \beta_{C}=0$, we conclude from Lemma 2.3 that

$$
0 \leq\left(D_{F}-d\left(B_{n} / 2\right)\right) \cdot\left(\beta_{C}-(n-1) \beta_{n}\right)=F \cdot C-d(n-1) .
$$

Lemma 2.5. Let $F$ be a divisor in $X$. Let $C$ be a smooth rational curve in $X$, and consider the $n$-th symmetric product $C^{(n)}=\operatorname{Hilb}^{n}(C) \subset X^{[n]}$. Then

(i) every line in $C^{(n)} \cong \mathbb{P}^{n}$ is homologous to $\beta_{C}-(n-1) \beta_{n}$;

(ii) $\left.\mathcal{O}_{X^{[n]}}\left(D_{F}\right)\right|_{C^{(n)}}=\mathcal{O}_{C^{(n)}}(C \cdot F)$ and $\left.\mathcal{O}_{X^{[n]}}\left(B_{n} / 2\right)\right|_{C^{(n)}}=\mathcal{O}_{C^{(n)}}(n-1)$. 
Proof. (i) Let $x_{1}, \ldots, x_{n-1} \in C$ be distinct, and put $\gamma=C+\left(x_{1}+\cdots+x_{n-1}\right)$. Then $\gamma$ is a line in the projective space $C^{(n)} \cong \mathbb{P}^{n}$. By Lemma 2.3,

$$
\gamma \sim \beta_{C}-(n-1) \beta_{n},
$$

where $\sim$ denotes homologous relation. So every line in $C^{(n)} \cong \mathbb{P}^{n}$ is homologous to the class $\beta_{C}-(n-1) \beta_{n}$.

(ii) Since $\left.\gamma \cdot D_{F}\right|_{C^{(n)}}=\gamma \cdot D_{F}=\left(\beta_{C}-(n-1) \beta_{n}\right) \cdot D_{F}=C \cdot F$, we get

$$
\left.\mathcal{O}_{X^{[n]}}\left(D_{F}\right)\right|_{C^{(n)}}=\mathcal{O}_{C^{(n)}}(C \cdot F) .
$$

Using a similar method, we obtain $\left.\mathcal{O}_{X^{[n]}}\left(B_{n} / 2\right)\right|_{C^{(n)}}=\mathcal{O}_{C^{(n)}}(n-1)$.

In the rest of the paper, we assume that $h^{1}\left(X, \mathcal{O}_{X}\right)=0$. Then

$$
\operatorname{Pic}\left(X^{[n]}\right) \cong \operatorname{Pic}(X) \oplus \mathbb{Z} \cdot\left(B_{n} / 2\right)
$$

by [Fogarty 1973]. Under this isomorphism, the divisor $D_{C} \in \operatorname{Pic}\left(X^{[n]}\right)$ corresponds to $C \in \operatorname{Pic}(X)$. Let $\left\{\alpha_{1}, \ldots, \alpha_{s}\right\}$ be a linear basis of $H^{2}(X)$. Then

$$
\left\{D_{\alpha_{1}}, \ldots, D_{\alpha_{s}}, B_{n}\right\}
$$

is a linear basis of $H^{2}\left(X^{[n]}\right)$. Represent $\alpha_{1}, \ldots, \alpha_{s}$ by real surfaces $C_{1}, \ldots, C_{s} \subset X$, respectively. Then a linear basis of $H_{2}\left(X^{[n]}\right)$ is given by

$$
\left\{\beta_{C_{1}}, \ldots, \beta_{C_{s}}, \beta_{n}\right\} \text {. }
$$

We are now ready to prove our main result in this paper.

Theorem 2.6. Let $n \geq 2$, and let the surface $X$ satisfy $h^{1}\left(X, \mathcal{O}_{X}\right)=0$. Assume that the nef cone of $X$ is the span of the divisors $F_{1}, \ldots, F_{t}$, and the cone $\operatorname{NE}(X)$ is the span of the curves $C_{1}, \ldots, C_{t}$ with $F_{i} \cdot C_{j}=\delta_{i, j}$ for all $i$ and $j$. Assume further that $\mathcal{O}_{X}\left((n-1) \sum_{i=1}^{t} F_{i}\right)$ is $(n-1)$-very ample. Then

(i) the nef cone of the Hilbert scheme $X^{[n]}$ is spanned by

$$
D_{F_{1}}, \ldots, D_{F_{t}},(n-1) \sum_{i=1}^{t} D_{F_{i}}-B_{n} / 2 ;
$$

(ii) the cone $\mathrm{NE}\left(X^{[n]}\right)$ is spanned by the classes

$$
\beta_{C_{1}}-(n-1) \beta_{n}, \ldots, \beta_{C_{t}}-(n-1) \beta_{n}, \beta_{n} .
$$

Proof. (i) For $1 \leq j \leq n$, let $p_{j}: X^{n} \rightarrow X$ be the projection to the $j$-th factor. Let $X^{(n)}$ be the $n$-th symmetric product of $X$, and let $v_{n}: X^{n} \rightarrow X^{(n)}$ be the quotient map. Let $\rho_{n}: X^{[n]} \rightarrow X^{(n)}$ be the Hilbert-Chow morphism sending an element 
$\xi \in X^{[n]}$ to its support (with multiplicities) in $X^{(n)}$. For each $F_{i}$, there exists a divisor $H_{i}$ on $X^{(n)}$ such that

$$
\rho_{n}^{*} H_{i}=D_{F_{i}}, \quad v_{n}^{*} H_{i}=\sum_{j=1}^{n} p_{j}^{*} F_{i} .
$$

It follows that since $F_{i}$ is nef, the divisor $D_{F_{i}}$ is nef as well. Since the line bundle $\mathcal{O}_{X}\left((n-1) \sum_{i=1}^{t} F_{i}\right)$ is $(n-1)$-very ample, we conclude from Lemma 2.2 that $(n-1) \sum_{i=1}^{t} D_{F_{i}}-B_{n} / 2$ is a nef divisor. Thus, the cone $\boldsymbol{C}_{1}$ spanned by the divisors in (2-19) is contained in the nef cone of $X^{[n]}$.

Conversely, assume that $D_{F}-d B_{n} / 2$ is a nef divisor on $X^{[n]}$. Let $F=\sum_{i=1}^{t} a_{i} F_{i}$. By Lemma 2.4, $d \geq 0$ and $F \cdot C \geq d(n-1)$ for every irreducible curve $C \subset X$. So

$$
a_{i}=F \cdot C_{i} \geq d(n-1)
$$

for every $i$. Now the nef divisor $D_{F}-d B_{n} / 2$ can be written as

$$
\sum_{i=1}^{t} a_{i} D_{F_{i}}-d B_{n} / 2=\sum_{i=1}^{t}\left(a_{i}-d(n-1)\right) D_{F_{i}}+d\left((n-1) \sum_{i=1}^{t} D_{F_{i}}-B_{n} / 2\right) .
$$

Therefore, $D_{F}-d B_{n} / 2 \in C_{1}$. It follows that $\boldsymbol{C}_{1}$ is the nef cone of $X^{[n]}$.

(ii) First of all, note that since the divisor $F_{i}$ is nef and $F_{i} \cdot C_{i}=1$, the curve $C_{i}$ contains at least one reduced irreducible component. So the curve $\beta_{C_{i}}-(n-1) \beta_{n}$ is well-defined, and the cone $\boldsymbol{C}_{2}$ spanned by the curves in (2-20) is contained in the cone $\operatorname{NE}\left(X^{[n]}\right)$. Conversely, assume that $\sum_{i=1}^{t} b_{i} \beta_{C_{i}}+c \beta_{n}$ is contained in $\operatorname{NE}\left(X^{[n]}\right)$. Then $\left(\sum_{i=1}^{t} b_{i} \beta_{C_{i}}+c \beta_{n}\right) \cdot H \geq 0$ for every nef divisor $H$ in $X^{[n]}$. Letting $H=D_{F_{i}}$, we get $b_{i} \geq 0$ for every $i$. Letting $H=(n-1) \sum_{i=1}^{t} D_{F_{i}}-B_{n} / 2$, we obtain $(n-1) \sum_{i=1}^{t} b_{i}+c \geq 0$. Therefore, we have

$$
\sum_{i=1}^{t} b_{i} \beta_{C_{i}}+c \beta_{n}=\sum_{i=1}^{t} b_{i}\left(\beta_{C_{i}}-(n-1) \beta_{n}\right)+\left((n-1) \sum_{i=1}^{t} b_{i}+c\right) \beta_{n} \in \boldsymbol{C}_{2} .
$$

It follows that $\boldsymbol{C}_{2}$ coincides with the cone $\mathrm{NE}\left(X^{[n]}\right)$.

Corollary 2.7. Under the same assumptions as in Theorem 2.6, if $\gamma$ is an irreducible curve in the Hilbert scheme $X^{[n]}$, then $\gamma$ is homologous to

$$
\sum_{i=1}^{t} b_{i}\left(\beta_{C_{i}}-(n-1) \beta_{n}\right)+c \beta_{n}
$$

for some nonnegative integers $b_{1}, \ldots, b_{t}, c$ not all equal to zero.

Proof. By Theorem 2.6 (ii), $b_{1}, \ldots, b_{t}, c$ are nonnegative and not all equal to 0 . Intersecting the curve $\gamma$ with the divisors $D_{F_{1}}, \ldots, D_{F_{t}}$ and $(n-1) \sum_{i=1}^{t} D_{F_{i}}-B_{n} / 2$, respectively, we see that $b_{1}, \ldots, b_{t}, c$ must be integers. 


\section{Application to Hirzebruch surfaces}

In this section, we apply Theorem 2.6 to the Hirzebruch surfaces and recover a result in [Bertram and Coskun 2013]. Then we study the curves in the Hilbert schemes of points on the Hirzebruch surfaces, which have the minimal degree. We compute their normal bundles, and prove that their moduli spaces are unobstructed.

Let $X$ denote the Hirzebruch surface $\mathbb{F}_{e}$ with $e \geq 0$. Let $f$ be a fiber of the ruling $\pi: X \rightarrow \mathbb{P}^{1}$, and $\sigma \subset X$ be a section of $\pi$ such that $\sigma^{2}=-e$. Then

$$
\operatorname{Pic}(X)=\mathbb{Z} \cdot \sigma \oplus \mathbb{Z} \cdot f
$$

It is well-known that $a \sigma+b f$ is nef if and only if $a \geq 0$ and $b \geq a e$. The following lemma was proved in [Beltrametti and Sommese 1993].

Lemma 3.1. $\mathcal{O}_{X}(a \sigma+b f)$ is $n$-very ample if and only if $a \geq n$ and $b \geq n+a e$.

Proposition 3.2. Let $n \geq 2$, and let $X$ be the Hirzebruch surface $\mathbb{F}_{e}$. Then

(i) the nef cone of the Hilbert scheme $X^{[n]}$ is spanned by

$$
D_{f}, \quad D_{\sigma}+e D_{f}, \quad(n-1) D_{\sigma}+(n-1)(1+e) D_{f}-B_{n} / 2 ;
$$

(ii) the cone $\mathrm{NE}\left(X^{[n]}\right)$ is spanned by the classes

$$
\beta_{\sigma}-(n-1) \beta_{n}, \quad \beta_{f}-(n-1) \beta_{n}, \quad \beta_{n} .
$$

Proof. The nef cone of $X$ is the span of $F_{1}=f$ and $F_{2}=\sigma+e f$, and the cone $\mathrm{NE}(X)$ is the span of $C_{1}=\sigma$ and $C_{2}=f$. Note that $F_{i} \cdot C_{j}=\delta_{i, j}$ for all $i$ and $j$. In addition, by Lemma 3.1, the line bundle $\mathcal{O}_{X}\left((n-1) F_{1}+(n-1) F_{2}\right)$ is $(n-1)$-very ample. Hence our proposition follows from Theorem 2.6.

Proposition 3.2 has been proved in [Bertram and Coskun 2013]. We now study the curves in $X^{[n]}$ whose homology classes are contained in the list (3-2). Let

$$
L_{n}=n \sigma+n(1+e) f .
$$

By Lemma 3.1, the line bundle $\mathcal{O}_{X}\left(L_{n}\right)$ is $n$-very ample. By Lemma 2.2, the divisor

$$
D_{L_{n}}-B_{n} / 2=n D_{\sigma}+n(1+e) D_{f}-B_{n} / 2
$$

in $X^{[n]}$ is very ample. Our next lemma characterizes the homology classes in (3-2).

Lemma 3.3. Let $\gamma$ be a curve in $X^{[n]}$ with $\gamma \cdot\left(n D_{\sigma}+n(1+e) D_{f}-B_{n} / 2\right)=1$. Then $\gamma$ is a smooth rational curve. Moreover, $\gamma \sim \beta_{n}, \beta_{f}-(n-1) \beta_{n}$ or $\beta_{\sigma}-(n-1) \beta_{n}$.

Proof. Since $n D_{\sigma}+n(1+e) D_{f}-B_{n} / 2$ is very ample, $\gamma$ is a smooth rational curve. By Corollary 2.7, $\gamma \sim a\left(\beta_{\sigma}-(n-1) \beta_{n}\right)+b\left(\beta_{f}-(n-1) \beta_{n}\right)+c \beta_{n}$ for some nonnegative integers $a, b, c$. Since $\gamma \cdot\left(n D_{\sigma}+n(1+e) D_{f}-B_{n} / 2\right)=1$, we obtain

$$
a+b+c=1 \text {. }
$$

Therefore, we have $\gamma \sim \beta_{n}, \beta_{f}-(n-1) \beta_{n}$ or $\beta_{\sigma}-(n-1) \beta_{n}$. 
The curves in $X^{[n]}$ homologous to $\beta_{n}$ have been classified in [Li et al. 2003]. In the rest of this section, we study the curves $\gamma \subset X^{[n]}$ which are homologous to $\beta_{f}-(n-1) \beta_{n}$ or $\beta_{\sigma}-(n-1) \beta_{n}$. By Lemma 3.1, the line bundle $\mathcal{O}_{X}\left(L_{n-1}\right)$ is $(n-1)$-very ample. So by (2-9), we have the morphism

$$
\varphi:=\varphi_{n}\left(\mathcal{O}_{X}\left(L_{n-1}\right)\right): X^{[n]} \rightarrow \mathbb{G}\left(H^{0}\left(X, \mathcal{O}_{X}\left(L_{n-1}\right)\right), n\right) .
$$

Lemma 3.4. Let $\gamma$ be an irreducible curve in $X^{[n]}$ satisfying

$$
\gamma \cdot\left((n-1) D_{\sigma}+(n-1)(1+e) D_{f}-B_{n} / 2\right)=0 .
$$

Then $\gamma \sim \beta_{f}-(n-1) \beta_{n}$ or $\beta_{\sigma}-(n-1) \beta_{n}$. Moreover, $\gamma$ is contracted by $\varphi$.

Proof. The first part of the lemma is proved by an argument similar to the proof of Lemma 3.3. For the second part, we notice from (2-10) that

$$
\begin{aligned}
(\mathfrak{P} \circ \varphi)^{*} \mathcal{H} & =\mathcal{O}_{X^{[n]}}\left(D_{L_{n-1}}-B_{n} / 2\right) \\
& =\mathcal{O}_{X^{[n]}}\left((n-1) D_{\sigma}+(n-1)(1+e) D_{f}-B_{n} / 2\right) .
\end{aligned}
$$

Therefore, the curve $\gamma$ is contracted by the morphism $\mathfrak{P} \circ \varphi$. Since $\mathfrak{P}$ is an embedding, the curve $\gamma$ is contracted by the morphism $\varphi$.

In the following, we fix a curve $\gamma \subset X^{[n]}$ homologous to $\beta_{f}-(n-1) \beta_{n}$ or $\beta_{\sigma}-(n-1) \beta_{n}$. Let $X^{(n)}$ be the $n$-th symmetric product of $X$ and $v_{n}: X^{n} \rightarrow X^{(n)}$ the quotient map. Let $\rho_{n}: X^{[n]} \rightarrow X^{(n)}$ be the Hilbert-Chow morphism sending an element $\xi \in X^{[n]}$ to its support (with multiplicities) in $X^{(n)}$. Let $p_{1}$ be the projection from $X^{n}$ to the first factor.

Definition 3.5. Define $C_{\gamma}$ to be the union of all the curves in $p_{1}\left(v_{n}^{-1}\left(\rho_{n}(\gamma)\right)\right)$.

Lemma 3.6. Let $\gamma \sim \beta_{f}-(n-1) \beta_{n}$ or $\beta_{\sigma}-(n-1) \beta_{n}$. Then $C_{\gamma} \sim \sigma$ or $f$.

Proof. First of all, we claim that $C_{\gamma} \neq \varnothing$. Indeed, if $C_{\gamma}=\varnothing$, then $p_{1}\left(v_{n}^{-1}\left(\rho_{n}(\gamma)\right)\right)$ is a finite set of points in $X$. Since the divisor $\sigma+(1+e) f$ is very ample, we can choose a smooth curve $F \in|\sigma+(1+e) f|$ such that $F \cap p_{1}\left(v_{n}^{-1}\left(\rho_{n}(\gamma)\right)\right)=\varnothing$. Since the elements of $\gamma$ are supported in $p_{1}\left(v_{n}^{-1}\left(\rho_{n}(\gamma)\right)\right)$, we must have $\gamma \cap D_{F}=\varnothing$. It follows that $\gamma \cdot D_{F}=0$. However, this contradicts $\gamma \cdot D_{F}=1$.

Next, assume that $C_{\gamma} \cdot(\sigma+(1+e) f) \geq 2$. Take a point $\xi \in \gamma$ and a smooth point $x \in C_{\gamma}$ such that $x \notin \operatorname{Supp}(\xi)$. Since $x \in C_{\gamma} \subset p_{1}\left(v_{n}^{-1}\left(\rho_{n}(\gamma)\right)\right)$, there exists $\xi_{x} \in \gamma$ such that $\rho_{n}\left(\xi_{x}\right)=n_{x} x+\eta_{x}$, where $n_{x} \geq 1, \eta_{x} \in X^{\left(n-n_{x}\right)}$ and $x \notin \operatorname{Supp}\left(\eta_{x}\right)$. Choose a smooth curve $F \in|\sigma+(1+e) f| \operatorname{missing} \operatorname{Supp}\left(\eta_{x}\right) \cup \operatorname{Supp}(\xi)$, passing through $x$, and intersecting $C_{\gamma}$ transversally. Then $F \cap C_{\gamma}$ is a finite set. Since $C_{\gamma} \cdot F \geq 2$, $F \cap C_{\gamma}$ contains one more point $y \neq x$. Hence there exists $\xi_{y} \in \gamma$ with $y \in \operatorname{Supp}\left(\xi_{y}\right)$. Thus $\xi_{x}, \xi_{y} \in \gamma \cap D_{F}$. Since $y \neq x, y \in F$ and $F$ misses $\operatorname{Supp}\left(\eta_{x}\right)$, we get

$$
y \notin\{x\} \cup \operatorname{Supp}\left(\eta_{x}\right)=\operatorname{Supp}\left(\xi_{x}\right) .
$$


So $\xi_{x} \neq \xi_{y}$. Since $\operatorname{Supp}(\xi) \cap F=\varnothing$, we have $\xi \notin D_{F}$. Since $\xi \in \gamma$ and $\gamma$ is a smooth rational curve, $\gamma$ is not contained in $D_{F}$. Therefore, $\gamma \cap D_{F}$ is a finite set of points. Since $\xi_{x}, \xi_{y} \in \gamma \cap D_{F}$ and $\xi_{x} \neq \xi_{y}$, we obtain $\gamma \cdot D_{F} \geq 2$, which contradicts $\gamma \cdot D_{F}=1$.

It follows that $C_{\gamma} \cdot(\sigma+(1+e) f)=1$. Since the cone $\mathrm{NE}\left(\mathbb{F}_{e}\right)$ is spanned by $\sigma$ and $f$, we conclude that $C_{\gamma} \sim \sigma$ or $f$.

By Lemma 3.6, $C_{\gamma} \sim \sigma$ or $f$. So $C_{\gamma}$ is a smooth rational curve, and

$$
\left.\mathcal{O}_{X}\left(L_{n-1}\right)\right|_{C_{\gamma}} \cong \mathcal{O}_{C_{\gamma}}(n-1) .
$$

Let $V_{C_{\gamma}} \subset H^{0}\left(X, \mathcal{O}_{X}\left(L_{n-1}\right)\right)$ be the image of the injection

$$
H^{0}\left(X, \mathcal{O}_{X}\left(L_{n-1}-C_{\gamma}\right)\right) \rightarrow H^{0}\left(X, \mathcal{O}_{X}\left(L_{n-1}\right)\right),
$$

which is induced by the exact sequence

$$
0 \rightarrow \mathcal{O}_{X}\left(L_{n-1}-C_{\gamma}\right) \rightarrow \mathcal{O}_{X}\left(L_{n-1}\right) \rightarrow \mathcal{O}_{C_{\gamma}}(n-1) \rightarrow 0 .
$$

Similarly, for $\xi \in \gamma$, let $V_{\xi} \subset H^{0}\left(X, \mathcal{O}_{X}\left(L_{n-1}\right)\right)$ be the image of the injection

$$
H^{0}\left(X, \mathcal{O}_{X}\left(L_{n-1}\right) \otimes I_{\xi}\right) \rightarrow H^{0}\left(X, \mathcal{O}_{X}\left(L_{n-1}\right)\right) .
$$

Since $\mathcal{O}_{X}\left(L_{n-1}\right)$ is $(n-1)$-very ample, we obtain

$$
\operatorname{dim} V_{\xi}=h^{0}\left(X, \mathcal{O}_{X}\left(L_{n-1}\right)\right)-h^{0}\left(\mathcal{O}_{\xi}\right)=h^{0}\left(X, \mathcal{O}_{X}\left(L_{n-1}\right)\right)-n .
$$

Since the curve $\gamma$ is contracted to a point by the morphism $\varphi$, the subspaces $V_{\xi}$ of $H^{0}\left(X, \mathcal{O}_{X}\left(L_{n-1}\right)\right)$ are independent of $\xi \in \gamma$. Set $V_{\gamma}=V_{\xi}$ where $\xi \in \gamma$.

Lemma 3.7. If $n \geq e+1$, then $V_{C_{\gamma}}=V_{\gamma}$.

Proof. Since $K_{X}=-2 \sigma-(2+e) f$, the divisor $L_{n-1}-C_{\gamma}-K_{X}$ is ample if $C_{\gamma}=\sigma$. Similarly, since $n \geq e+1, L_{n-1}-C_{\gamma}-K_{X}$ is ample if $C_{\gamma}=f$. By the Kodaira vanishing theorem, $H^{1}\left(X, \mathcal{O}_{X}\left(L_{n-1}-C_{\gamma}\right)\right)=0$. So we see from (3-3) that

$$
\operatorname{dim} V_{C_{\gamma}}=h^{0}\left(X, \mathcal{O}_{X}\left(L_{n-1}\right)\right)-h^{0}\left(C_{\gamma}, \mathcal{O}_{C_{\gamma}}(n-1)\right)=h^{0}\left(X, \mathcal{O}_{X}\left(L_{n-1}\right)\right)-n .
$$

In view of (3-4), we conclude that

$$
\operatorname{dim} V_{C_{\gamma}}=\operatorname{dim} V_{\xi}=\operatorname{dim} V_{\gamma} .
$$

Thus, to prove our lemma, it remains to prove that $V_{\gamma} \subset V_{C_{\gamma}}$. Indeed, let $f \in V_{\gamma}$ be a section. Let $x \in C_{\gamma}$. Since $C_{\gamma} \subset p_{1}\left(v_{n}^{-1}\left(\rho_{n}(\gamma)\right)\right)$, there exists $\xi \in \gamma$ such that $x \in \operatorname{Supp}(\xi)$. Since $V_{\gamma}=V_{\xi}, f$ vanishes at every point in $\operatorname{Supp}(\xi)$. In particular, $f$ vanishes at $x$. Hence, $f$ vanishes along the smooth curve $C_{\gamma}$. Therefore, $f \in V_{C_{\gamma}}$. It follows that $V_{\gamma} \subset V_{C_{\gamma}}$. 
Proposition 3.8. Let $n \geq \max (2, e+1)$. Then a curve $\gamma \subset X^{[n]}$ is homologous to $\beta_{f}-(n-1) \beta_{n}$ or $\beta_{\sigma}-(n-1) \beta_{n}$ if and only if there is a curve $C \subset X$ such that $C \sim \sigma$ or $f$, and that $\gamma$ is a line in $\operatorname{Hilb}^{n}(C) \subset X^{[n]}$. Moreover, the curve $C$ is uniquely determined by the curve $\gamma$.

Proof. The "if" part of the proposition follows from Lemma 2.5 (i). To prove the "only if" part, let $\gamma \sim \beta_{f}-(n-1) \beta_{n}$ or $\beta_{\sigma}-(n-1) \beta_{n}$. By Lemma 3.6, $C:=C_{\gamma} \sim \sigma$ or $f$. Fix a section $f_{0} \in H^{0}\left(X, \mathcal{O}_{X}(C)\right)$ whose zero locus is $C$. Let $\xi \in \gamma$. Since $\sigma+e f$ is basepoint-free, so is the divisor $L_{n-1}-C$. Thus there exists $f_{1} \in H^{0}\left(X, \mathcal{O}_{X}\left(L_{n-1}-C\right)\right)$ such that $f_{1}$ does not vanish at any point in $\operatorname{Supp}(\xi)$. Now, $f_{0} \otimes f_{1} \in V_{C}$. By Lemma 3.7, $f_{0} \otimes f_{1} \in V_{\gamma}=V_{\xi}$. Since $f_{1}$ does not vanish at any point in $\operatorname{Supp}(\xi), f_{0}$ vanishes at $\xi$. Hence, $\xi$ is a closed subscheme of $C$. It follows that $\gamma \subset \operatorname{Hilb}^{n}(C)$.

To show that $\gamma$ is a line in $\operatorname{Hilb}^{n}(C) \subset X^{[n]}$, let $F=\sigma+(e+1) f$. By Lemma 2.5 (ii), $\left.\mathcal{O}_{X^{[n]}}\left(D_{F}\right)\right|_{\operatorname{Hilb}^{n}(C)}=\mathcal{O}_{\operatorname{Hilb}^{n}(C)}(1)$. So viewing $\gamma$ as a curve in $\operatorname{Hilb}^{n}(C)$, we obtain

$$
\gamma \cdot c_{1}\left(\mathcal{O}_{\operatorname{Hilb}^{n}(C)}(1)\right)=\gamma \cdot D_{F}=1 .
$$

Therefore, $\gamma$ is a line in $\operatorname{Hilb}^{n}(C) \subset X^{[n]}$.

Finally, the uniqueness of $C$ follows from the observation that if $\xi \in X^{[n]}$ and $n \geq 2$, then $\xi$ is contained in at most one curve $C \subset X$ with $C \sim \sigma$ or $f$.

Next, we determine the normal bundle of a curve $\gamma$ in $X^{[n]}$ homologous to $\beta_{f}-(n-1) \beta_{n}$. By Proposition 3.8, there exists a unique fiber $f_{\gamma}$ in $X$ such that $\gamma$ is a line in the $n$-th symmetric product $f_{\gamma}^{(n)}=\operatorname{Hilb}^{n}\left(f_{\gamma}\right) \subset X^{[n]}$. In particular,

$$
N_{\gamma \subset f_{\gamma}^{(n)}} \cong \mathcal{O}_{\gamma}(1)^{\oplus(n-1)} \text {. }
$$

So we have the following exact sequence of normal bundles:

$$
\left.0 \rightarrow \mathcal{O}_{\gamma}(1)^{\oplus(n-1)} \rightarrow N_{\gamma \subset X^{[n]}} \rightarrow N_{f_{\gamma}^{(n)} \subset X^{[n]}}\right|_{\gamma} \rightarrow 0 .
$$

Lemma 3.9. Let $n \geq \max (2, e+1)$. Let $\gamma \subset X^{[n]}$ be a curve homologous to $\beta_{f}-(n-1) \beta_{n}$, and $f_{\gamma}$ be the unique fiber in $X$ such that $\gamma$ is a line in $f_{\gamma}^{(n)}$. Then

(i) $N_{f_{\gamma}^{(n)} \subset X^{[n]}} \cong \mathcal{O}_{f_{\gamma}^{(n)}} \oplus \mathcal{O}_{f_{\gamma}^{(n)}}(-1)^{\oplus(n-1)}$;

(ii) $N_{\gamma \subset X^{[n]}} \cong \mathcal{O}_{\gamma}(1)^{\oplus(n-1)} \oplus \mathcal{O}_{\gamma} \oplus \mathcal{O}_{\gamma}(-1)^{\oplus(n-1)}$.

Proof. (i) First of all, let $C \subset X$ be a smooth irreducible curve. Let $\pi_{n}$ and $q_{n}$ be the projections of $X^{[n]} \times X$ to $X^{[n]}$ and $X$ respectively. Recall the universal codimension-2 subscheme $\mathcal{Z}_{n} \subset X^{[n]} \times X$ from (2-11). By the results in [Altman et al. 1977], we have the isomorphism

$$
\left.N_{C^{(n)} \subset X^{[n]}} \cong \pi_{n *}\left(\left.q_{n}^{*} \mathcal{O}_{X}(C)\right|_{\mathcal{Z}_{n}}\right)\right|_{C^{(n)}} .
$$


Let $\widetilde{\mathcal{Z}}_{n}$ be the universal subscheme in $C^{(n)} \times C$. Then we obtain

$$
\left.\pi_{n *}\left(\left.q_{n}^{*} \mathcal{O}_{X}(C)\right|_{\mathcal{Z}_{n}}\right)\right|_{C^{(n)}} \cong \tilde{\pi}_{n *}\left(\left.\tilde{q}_{n}^{*}\left(\left.\mathcal{O}_{X}(C)\right|_{C}\right)\right|_{\tilde{Z}_{n}}\right),
$$

where $\tilde{\pi}_{n}$ and $\tilde{q}_{n}$ are the projections from $C^{(n)} \times C$ to $C^{(n)}$ and $C$, respectively. So

$$
N_{C^{(n)} \subset X^{[n]}} \cong \tilde{\pi}_{n *}\left(\left.\tilde{q}_{n}^{*}\left(\left.\mathcal{O}_{X}(C)\right|_{C}\right)\right|_{\tilde{\mathcal{Z}}_{n}}\right) .
$$

Replacing $C$ in (3-6) by $f_{\gamma}$, we get $N_{f_{\gamma}^{(n)} \subset X^{[n]}} \cong \tilde{\pi}_{n *} \mathcal{O}_{\widetilde{Z}_{n}}$. It is known that $\widetilde{\mathcal{Z}}_{n} \subset f_{\gamma}^{(n)} \times f_{\gamma} \cong \mathbb{P}^{n} \times \mathbb{P}^{1}$ is defined by the equation

$$
a_{0} U^{n}+a_{1} U^{n-1} V+\cdots+a_{n} V^{n}=0,
$$

where $a_{0}, a_{1}, \ldots, a_{n}$ and $U, V$ are the homogeneous coordinates on $\mathbb{P}^{n}$ and $\mathbb{P}^{1}$, respectively. So the line bundle $\mathcal{O}_{f_{\gamma}^{(n)} \times f_{\gamma}}\left(\widetilde{\mathcal{Z}}_{n}\right) \cong \mathcal{O}_{\mathbb{P}^{n} \times \mathbb{P}^{1}}\left(\widetilde{\mathcal{Z}}_{n}\right)$ is of type $(1, n)$ in

$$
\operatorname{Pic}\left(f_{\gamma}^{(n)} \times f_{\gamma}\right) \cong \operatorname{Pic}\left(\mathbb{P}^{n} \times \mathbb{P}^{1}\right) \cong \mathbb{Z} \oplus \mathbb{Z}
$$

Applying $\tilde{\pi}_{n *}$ to the exact sequence

$$
0 \rightarrow \mathcal{O}_{f_{\gamma}^{(n)} \times f_{\gamma}}\left(-\widetilde{\mathcal{Z}}_{n}\right) \rightarrow \mathcal{O}_{f_{\gamma}^{(n)} \times f_{\gamma}} \rightarrow \mathcal{O}_{\widetilde{Z}_{n}} \rightarrow 0
$$

we obtain the exact sequence

$$
0 \rightarrow \mathcal{O}_{f_{\gamma}^{(n)}} \rightarrow \tilde{\pi}_{n *} \mathcal{O}_{\widetilde{\mathcal{Z}}_{n}} \rightarrow \mathcal{O}_{f_{\gamma}^{(n)}}(-1)^{\oplus(n-1)} \rightarrow 0 .
$$

This exact sequence splits. Thus, $\tilde{\pi}_{n *} \mathcal{O}_{\widetilde{\mathcal{Z}}_{n}} \cong \mathcal{O}_{f_{\gamma}^{(n)}} \oplus \mathcal{O}_{f_{\gamma}^{(n)}}(-1)^{\oplus(n-1)}$. Hence

$$
N_{f_{\gamma}^{(n)} \subset X^{[n]}} \cong \tilde{\pi}_{n *} \mathcal{O}_{\widetilde{\mathcal{Z}}_{n}} \cong \mathcal{O}_{f_{\gamma}^{(n)}} \oplus \mathcal{O}_{f_{\gamma}^{(n)}}(-1)^{\oplus(n-1)} .
$$

(ii) By (i) and (3-5), we obtain the exact sequence

$$
0 \rightarrow \mathcal{O}_{\gamma}(1)^{\oplus(n-1)} \rightarrow N_{\gamma \subset X^{[n]}} \rightarrow \mathcal{O}_{\gamma} \oplus \mathcal{O}_{\gamma}(-1)^{\oplus(n-1)} \rightarrow 0 .
$$

Since this exact sequence splits, the proof of (ii) is complete.

Now we determine the normal bundle of a curve $\gamma$ in $X^{[n]}$ homologous to $\beta_{\sigma}-(n-1) \beta_{n}$. Recall that the Hirzebruch surfaces $\mathbb{F}_{e}$ are deformation equivalent to either $\mathbb{F}_{0}$ or $\mathbb{F}_{1}$. If $X=\mathbb{F}_{0}=\mathbb{P}^{1} \times \mathbb{P}^{1}$, then $\sigma$ is a fiber of one of the two rulings on $X$, so the normal bundle of a curve $\gamma$ in $X^{[n]}$ homologous to $\beta_{\sigma}-(n-1) \beta_{n}$ has been computed by Lemma 3.9 (ii). In the following, we concentrate on $X=\mathbb{F}_{1}$, which is the blowup of the projective plane at a point.

Lemma 3.10. Let $n \geq 2$ and $X=\mathbb{F}_{1}$. Let $\gamma \subset X^{[n]}$ be a curve homologous to $\beta_{\sigma}-(n-1) \beta_{n}$. Then, $N_{\sigma^{(n)} \subset X^{[n]}} \cong \mathcal{O}_{\sigma^{(n)}}(-1)^{\oplus n}$ and

$$
N_{\gamma \subset X^{[n]}} \cong \mathcal{O}_{\gamma}(1)^{\oplus(n-1)} \oplus \mathcal{O}_{\gamma}(-1)^{\oplus n} .
$$


Proof. The proof is similar to that of Lemma 3.9, so we adopt the notation in the proof of Lemma 3.9. Since $\sigma^{2}=-1$, we have $\left.\mathcal{O}_{X}(\sigma)\right|_{\sigma} \cong \mathcal{O}_{\sigma}(-1)$. Replacing the curve $C$ in (3-6) by $\sigma$, we conclude that

$$
N_{\sigma^{(n)} \subset X^{[n]}} \cong \tilde{\pi}_{n *}\left(\left.\tilde{q}_{n}^{*} \mathcal{O}_{\sigma}(-1)\right|_{\tilde{\mathcal{Z}}_{n}}\right) .
$$

Applying $\tilde{\pi}_{n *}$ to the exact sequence

$$
\left.0 \rightarrow \tilde{q}_{n}^{*} \mathcal{O}_{\sigma}(-1) \otimes \mathcal{O}_{\sigma^{(n)} \times \sigma}\left(-\widetilde{\mathcal{Z}}_{n}\right) \rightarrow \tilde{q}_{n}^{*} \mathcal{O}_{\sigma}(-1) \rightarrow \tilde{q}_{n}^{*} \mathcal{O}_{\sigma}(-1)\right|_{\tilde{Z}_{n}} \rightarrow 0,
$$

we obtain $\tilde{\pi}_{n *}\left(\tilde{q}_{n}^{*} \mathcal{O}_{\sigma}(-1) \mid \widetilde{\mathcal{Z}}_{n}\right) \cong \mathcal{O}_{\sigma^{(n)}}(-1)^{\oplus n}$. Therefore, we get

$$
N_{\sigma^{(n)} \subset X^{[n]}} \cong \mathcal{O}_{\sigma^{(n)}}(-1)^{\oplus n} .
$$

By Proposition 3.8, $\gamma$ is a line in $\sigma^{(n)} \cong \mathbb{P}^{n}$. Using the exact sequence

$$
\left.0 \rightarrow \mathcal{O}_{\gamma}(1)^{\oplus(n-1)} \rightarrow N_{\gamma \subset X^{[n]}} \rightarrow N_{\sigma^{(n)} \subset X^{[n]}}\right|_{\gamma} \rightarrow 0
$$

and (3-8), we see that $N_{\gamma \subset X^{[n]}} \cong \mathcal{O}_{\gamma}(1)^{\oplus(n-1)} \oplus \mathcal{O}_{\gamma}(-1)^{\oplus n}$.

Theorem 3.11. Let $X$ be the Hirzebruch surface $\mathbb{F}_{e}$ with $e \geq 0$, let $f$ be a fiber of the ruling on $X$, and let $\sigma$ be a section to the ruling with $\sigma^{2}=-e$.

(i) If $n \geq \max (2, e+1)$, then the moduli space $\mathfrak{M}\left(\beta_{f}-(n-1) \beta_{n}\right)$ of all the curves in $X^{[n]}$ homologous to $\beta_{f}-(n-1) \beta_{n}$ is irreducible and unobstructed, i.e., is smooth with the expected dimension.

(ii) If $e=1$ and $n \geq 2$, then the moduli space $\mathfrak{M}\left(\beta_{\sigma}-(n-1) \beta_{n}\right)$ of all the curves in $X^{[n]}$ homologous to $\beta_{\sigma}-(n-1) \beta_{n}$ is irreducible and unobstructed.

Proof. Under the assumptions of (i) and (ii), we see from Lemma 3.9 (ii) and (3-7) that $H^{1}\left(\gamma, N_{\gamma \subset X^{[n]}}\right)=0$ if $\gamma$ is a curve homologous to either $\beta_{f}-(n-1) \beta_{n}$ or $\beta_{\sigma}-(n-1) \beta_{n}$. This implies that the moduli spaces $\mathfrak{M}\left(\beta_{f}-(n-1) \beta_{n}\right)$ and $\mathfrak{M}\left(\beta_{\sigma}-(n-1) \beta_{n}\right)$ are unobstructed. By Proposition 3.8, $\mathfrak{M}\left(\beta_{f}-(n-1) \beta_{n}\right)$ is irreducible with dimension $2 n-1$, and $\mathfrak{M}\left(\beta_{\sigma}-(n-1) \beta_{n}\right)$ is irreducible with dimension $2 n-2$.

By (2-10), the composition $\mathfrak{P} \circ \varphi_{n}\left(L_{n}\right): X^{[n]} \rightarrow \mathbb{P}^{N}$ (for a suitable positive integer $N$ ) is the embedding associated to the very ample divisor

$$
D_{L_{n}}-B_{n} / 2=n D_{\sigma}+n(1+e) D_{f}-B_{n} / 2 \text {. }
$$

By Lemma 3.3, a curve $\gamma \subset X^{[n]}$ is mapped to a line in $\mathbb{P}^{N}$ if and only if $\gamma$ is homologous to $\beta_{n}, \beta_{f}-(n-1) \beta_{n}$ or $\beta_{\sigma}-(n-1) \beta_{n}$. Therefore, regarding $X^{[n]}$ as a closed subvariety of $\mathbb{P}^{N}$, then the Hilbert scheme of lines in $X^{[n]}$ is the disjoint union of $\mathfrak{M}\left(\beta_{n}\right), \mathfrak{M}\left(\beta_{f}-(n-1) \beta_{n}\right)$ and $\mathfrak{M}\left(\beta_{\sigma}-(n-1) \beta_{n}\right)$. 


\section{Acknowledgment}

The authors thank the referees for carefully reading the manuscript and for providing valuable suggestions which have greatly improved the exposition of the paper.

\section{References}

[Altman et al. 1977] A. B. Altman, A. Iarrobino, and S. L. Kleiman, "Irreducibility of the compactified Jacobian", pp. 1-12 in Real and complex singularities (Oslo, 1976), edited by P. Holm, Sijthoff and Noordhoff, Alphen aan den Rijn, 1977. MR 0498546 Zbl 0415.14014

[Arcara et al. 2013] D. Arcara, A. Bertram, I. Coskun, and J. Huizenga, "The minimal model program for the Hilbert scheme of points on $\mathbb{P}^{2}$ and Bridgeland stability”, Adv. Math. 235 (2013), 580-626. MR 3010070 Zbl 1267.14023

[Beltrametti and Sommese 1991] M. C. Beltrametti and A. J. Sommese, "Zero cycles and $k$ th order embeddings of smooth projective surfaces", pp. 33-48 in Problems in the theory of surfaces and their classification (Cortona, 1988), edited by F. Catanese et al., Symposia Mathematica 32, Academic Press, London, 1991. MR 1273371 Zbl 0824.00026

[Beltrametti and Sommese 1993] M. C. Beltrametti and A. J. Sommese, "On the preservation of $k$-very ampleness under adjunction”, Math. Z. 212:2 (1993), 257-283. MR 1202811 Zbl 0806.14015

[Bertram and Coskun 2013] A. Bertram and I. Coskun, "The birational geometry of the Hilbert scheme of points on surfaces", pp. 15-55 in Birational geometry, rational curves, and arithmetic, edited by F. Bogomolov et al., Springer, New York, NY, 2013. MR 3114922 Zbl 1273.14032

[Bolognese et al. 2015] B. Bolognese, J. Huizenga, Y. Lin, E. Riedl, B. Schmidt, M. Woolf, and X. Zhao, "Nef cones of Hilbert schemes of points on surfaces", preprint, 2015. arXiv 1509.04722

[Catanese and Gottsche 1990] F. Catanese and L. Gottsche, " $d$-very-ample line bundles and embeddings of Hilbert schemes of 0-cycles", Manuscripta Math. 68:3 (1990), 337-341. MR 1065935 Zbl 0729.14006

[Cheah 1998] J. Cheah, "Cellular decompositions for nested Hilbert schemes of points", Pacific J. Math. 183:1 (1998), 39-90. MR 1616606 Zbl 0904.14001

[Ellingsrud and Strømme 1998] G. Ellingsrud and S. A. Strømme, "An intersection number for the punctual Hilbert scheme of a surface", Trans. Amer. Math. Soc. 350:6 (1998), 2547-2552. MR 1432198 Zbl 0893.14001

[Fogarty 1968] J. Fogarty, "Algebraic families on an algebraic surface", Amer. J. Math. 90 (1968), 511-521. MR 0237496 Zbl 0176.18401

[Fogarty 1973] J. Fogarty, "Algebraic families on an algebraic surface, II: The Picard scheme of the punctual Hilbert scheme”, Amer. J. Math. 95 (1973), 660-687. MR 0335512 Zbl 0299.14020

[Grojnowski 1996] I. Grojnowski, "Instantons and affine algebras, I: The Hilbert scheme and vertex operators”, Math. Res. Lett. 3:2 (1996), 275-291. MR 1386846 Zbl 0879.17011

[Iarrobino 1977] A. A. Iarrobino, Punctual Hilbert schemes, Memoirs of the American Mathematical Society 10:188, American Mathematical Society, Providence, RI, 1977. MR 0485867 Zbl 0355.14001

[Li et al. 2003] W.-P. Li, Z. Qin, and Q. Zhang, "Curves in the Hilbert schemes of points on surfaces", pp. 89-96 in Vector bundles and representation theory (Columbia, MO, 2002), edited by S. D. Cutkosky et al., Contemporary Mathematics 322, American Mathematical Society, Providence, RI, 2003. MR 1987741 Zbl 1057.14012 
[Nakajima 1997] H. Nakajima, "Heisenberg algebra and Hilbert schemes of points on projective surfaces", Ann. of Math. (2) 145:2 (1997), 379-388. MR 1441880 Zbl 0915.14001

[Tikhomirov 1994] A. S. Tikhomirov, "Standard bundles on a Hilbert scheme of points on a surface", pp. 183-203 in Algebraic geometry and its applications (Yaroslavl, 1992), edited by A. Tikhomirov and A. Tyurin, Aspects of Mathematics E25, Vieweg, Braunschweig, 1994. MR 1282029 Zbl 0819.14003

Received October 18, 2015. Revised January 23, 2016.

\section{ZHENBO QIN}

DEPARTMENT OF MATHEMATICS

UNIVERSITY OF MISSOURI

COLUMBia, MO 65211

UNITED STATES

qinz@missouri.edu

YUPING TU

SCHOOL OF MATHEMATICS AND STATISTICS

WUHAN UNIVERSITY, WUHAN

430072 HUBEI

CHINA

yuping_tu@126.com 


\title{
PACIFIC JOURNAL OF MATHEMATICS
}

Founded in 1951 by E. F. Beckenbach (1906-1982) and F. Wolf (1904-1989)

$$
\text { msp.org/pjm }
$$

\section{EDITORS}

\author{
Don Blasius (Managing Editor) \\ Department of Mathematics \\ University of California \\ Los Angeles, CA 90095-1555 \\ blasius@math.ucla.edu
}

\author{
Paul Balmer \\ Department of Mathematics \\ University of California \\ Los Angeles, CA 90095-1555 \\ balmer@math.ucla.edu \\ Robert Finn \\ Department of Mathematics \\ Stanford University \\ Stanford, CA 94305-2125 \\ finn@math.stanford.edu \\ Sorin Popa \\ Department of Mathematics \\ University of California \\ Los Angeles, CA 90095-1555 \\ popa@math.ucla.edu
}

\author{
Vyjayanthi Chari \\ Department of Mathematics \\ University of California \\ Riverside, CA 92521-0135 \\ chari@math.ucr.edu \\ Kefeng Liu \\ Department of Mathematics \\ University of California \\ Los Angeles, CA 90095-1555 \\ liu@math.ucla.edu \\ Igor Pak \\ Department of Mathematics \\ University of California \\ Los Angeles, CA 90095-1555 \\ pak.pjm@gmail.com \\ Paul Yang \\ Department of Mathematics \\ Princeton University \\ Princeton NJ 08544-1000 \\ yang@math.princeton.edu
}

\section{PRODUCTION}

Silvio Levy, Scientific Editor, production@msp.org

\section{SUPPORTING INSTITUTIONS}

ACADEMIA SINICA, TAIPEI

CALIFORNIA INST. OF TECHNOLOGY

STANFORD UNIVERSITY

UNIV. OF BRITISH COLUMBIA

UNIV. OF CALIFORNIA, BERKELEY

UNIV. OF CALIFORNIA, DAVIS

UNIV. OF CALIFORNIA, LOS ANGELES

UNIV. OF CALIFORNIA, RIVERSIDE

UNIV. OF CALIFORNIA, SAN DIEGO

UNIV. OF CALIF., SANTA BARBARA
KEIO UNIVERSITY

MATH. SCIENCES RESEARCH INSTITUTE

NEW MEXICO STATE UNIV.

OREGON STATE UNIV.
Daryl Cooper

Department of Mathematics

University of California

Santa Barbara, CA 93106-3080 cooper@math.ucsb.edu

Jiang-Hua Lu

Department of Mathematics

The University of Hong Kong

Pokfulam Rd., Hong Kong

jhlu@maths.hku.hk

$$
\text { Jie Qing }
$$

Department of Mathematics

University of California

Santa Cruz, CA 95064

qing@ cats.ucsc.edu

\author{
UNIV. OF CALIF., SANTA CRUZ \\ UNIV. OF MONTANA \\ UNIV. OF OREGON \\ UNIV. OF SOUTHERN CALIFORNIA \\ UNIV. OF UTAH \\ UNIV. OF WASHINGTON \\ WASHINGTON STATE UNIVERSITY
}

These supporting institutions contribute to the cost of publication of this Journal, but they are not owners or publishers and have no responsibility for its contents or policies.

See inside back cover or msp.org/pjm for submission instructions.

The subscription price for 2016 is US $\$ 440 /$ year for the electronic version, and \$600/year for print and electronic.

Subscriptions, requests for back issues and changes of subscriber address should be sent to Pacific Journal of Mathematics, P.O. Box 4163, Berkeley, CA 94704-0163, U.S.A. The Pacific Journal of Mathematics is indexed by Mathematical Reviews, Zentralblatt MATH, PASCAL CNRS Index, Referativnyi Zhurnal, Current Mathematical Publications and Web of Knowledge (Science Citation Index).

The Pacific Journal of Mathematics (ISSN 0030-8730) at the University of California, c/o Department of Mathematics, 798 Evans Hall \#3840, Berkeley, CA 94720-3840, is published twelve times a year. Periodical rate postage paid at Berkeley, CA 94704, and additional mailing offices. POSTMASTER: send address changes to Pacific Journal of Mathematics, P.O. Box 4163, Berkeley, CA 94704-0163.

PJM peer review and production are managed by EditFLOW ${ }^{\circledR}$ from Mathematical Sciences Publishers.

PUBLISHED BY

\section{I. mathematical sciences publishers}

nonprofit scientific publishing

http://msp.org/

(C) 2016 Mathematical Sciences Publishers 


\section{PACIFIC JOURNAL OF MATHEMATICS}

Volume $284 \quad$ No. $2 \quad$ October 2016

Spherical CR Dehn surgeries

Miguel ACOsta

Degenerate flag varieties and Schubert varieties: a characteristic free approach

283

Giovanni Cerulli Irelli, Martina LANini and Peter

LITTELMANN

Solitons for the inverse mean curvature flow

Gregory Drugan, HoJoo LEE and GLEN WHEELER

Bergman theory of certain generalized Hartogs triangles

\section{LUKE D. EDHOLM}

Transference of certain maximal Hilbert transforms on the torus

DASHAN FAN, HUOXIONG WU and FAYOU ZHAO

The Turaev and Thurston norms

STEFAN FriedL, DANIEL S. Silver and Susan G. WiLLiams

A note on nonunital absorbing extensions

JAMES GABE

On nonradial singular solutions of supercritical biharmonic equations

Zongming GuO, JUNCHENG WeI and Wen YANG

Natural commuting of vanishing cycles and the Verdier dual

\section{DAVID B. MASSEY}

The nef cones of and minimal-degree curves in the Hilbert schemes of points on certain surfaces

ZHENBo QIN and YUPING TU

Smooth approximation of conic Kähler metric with lower Ricci curvature bound

\section{LIANGMING SHEN}

Maps from the enveloping algebra of the positive Witt algebra to regular algebras

Susan J. SierRa and Chelsea Walton 